\title{
Surgical microwave ablation for multifocal non-resectable liver metastases: a single institution experience treating five or more liver lesions
}

\author{
Jillian K. Wothe ${ }^{1 \wedge}$, Kendall R. McEachron ${ }^{1}$, Schelomo Marmor ${ }^{1}$, Jacob S. Ankeny ${ }^{1}$, \\ Christopher J. LaRocca ${ }^{1}$, Benjamin Spilseth ${ }^{2}$, Robben Schat ${ }^{2}$, Eric H. Jensen ${ }^{1}$ \\ ${ }^{1}$ Division of Oncology, Department of Surgery, University of Minnesota Medical School, Minneapolis, MN, USA; ${ }^{2}$ Department of Diagnostic \\ Radiology, University of Minnesota Medical School, Minneapolis, MN, USA \\ Contributions: (I) Conception and design: EH Jensen, JS Ankeny, JK Wothe; (II) Administrative support: EH Jensen; (III) Provision of study materials \\ or patients: EH Jensen; (IV) Collection and assembly of data: JK Wothe; (V) Data analysis and interpretation: JK Wothe, S Marmor; (VI) Manuscript \\ writing: All authors; (VII) Final approval of manuscript: All authors. \\ Correspondence to: Eric H. Jensen, MD, FACS. Division of Oncology, Department of Surgery, University of Minnesota Medical School, 420 Delaware \\ Street SE, MMC 195, Minneapolis, MN 55455, USA. Email: jense893@umn.edu.
}

\begin{abstract}
Background: Thermal ablation is an effective treatment for patients with metastatic colon and rectal cancer and allows surgeons to offer curative intent therapy to patients who are otherwise not candidates for resection. We aimed to report outcomes of a single institution experience using microwave ablation (MWA) with or without resection to treat five or more liver metastases.

Methods: In this retrospective cohort study, the University of Minnesota Division of Surgical Oncology liver surgery database was queried to identify all patients who underwent thermal ablation of five or more colorectal liver metastases (CRLM) between 2012-2018. We reviewed patient, disease, and tumor characteristics and measured local, intrahepatic, and extrahepatic recurrence (EHR) rates. We also calculated median overall survival (OS) and disease-free survival (DFS).

Results: Ten patients identified had five or more (range, 5-18) tumors ablated with or without combined liver and bowel resection. Median age was 50, and most patients were male (70\%) and Caucasian (90\%). Four patients received ablation alone (5-12 lesions), while six had combined resection and ablation (5-18 lesions). Ablation was performed laparoscopically in six patients, and four had ablations without resection. All patients received pre- and post-operative chemotherapy. A median of 7 tumors were ablated per patient. Median follow-up was 2.3 years. Among 75 tumors ablated, ablation site recurrence (ASR) (within $1 \mathrm{~cm}$ of ablation site) was seen in three with a per-lesion recurrence rate of $4 \%$. Intrahepatic recurrence (IHR) occurred in $6(60 \%)$ patients and EHR in $1(10 \%)$. Five patients underwent retreatment of IHR during follow-up. Median OS was 3 years and DFS was 7.1 months. At the time of last follow up, 6 patients were disease-free.

Conclusions: Thermal ablation can provide acceptable DFS and OS, even with high volume metastatic colorectal cancers. Future efforts should be focused on defining selection criteria for those most likely to benefit from this aggressive approach.
\end{abstract}

Keywords: Colorectal cancer; thermal ablation; liver metastases; microwave ablation (MWA)

Submitted Apr 17, 2021. Accepted for publication Sep 29, 2021.

doi: 10.21037/jgo-21-203

View this article at: https://dx.doi.org/10.21037/jgo-21-203

\footnotetext{
$\wedge$ ORCID: 0000-0003-2730-7638.
} 


\section{Introduction}

Colorectal cancer is the fourth most common cancer diagnosed in the United States, affecting more than a million Americans in 2017 (1). It is the 2nd leading cause of cancer related deaths, in part due to its propensity for metastasis (2). The most common extra-lymphatic site for metastasis is the liver, with approximately $30 \%$ of patients developing colorectal liver metastases (CRLM) (3). When the metastases are confined to the liver, surgical resection can be curative (4). However, due to anatomic limitations, the number or size of tumors, and other patient-related factors, a large percentage of hepatic metastases are considered unresectable and thus require alternative therapy (5). This can be via systemic chemotherapy, or liver directed therapy (5).

Recently, thermal ablation techniques have been increasingly used for the treatment of unresectable CRLM (6). While there is some debate over which thermal ablation method is superior, microwave ablation (MWA) has been theorized as having potential for larger ablation volumes, shorter procedure times, and less tissue trauma when compared to other methods (6). Other studies have reported the safety and efficacy of MWA, including decreased length of stay, comparable complication rates, and acceptable survival when compared to surgical resection in properly selected patients $(7,8)$. MWA has thus become increasingly used to treat otherwise unresectable cases of CRLM.

While percutaneous thermal ablation has good outcomes, some patients require a surgical approach to ablation due to anatomy and location of metastases (9). Laparoscopic or open ultrasound-guided approaches provide superior exposure of the liver and higher accuracy compared to percutaneous methods (9). In patients with hepatocellular carcinoma, Eun et al. compared laparoscopic with percutaneous radiofrequency ablation and found superior survival in those treated laparoscopically (9). There is need for more studies examining the safety and efficacy of laparoscopic ablation for CRLM, especially in patients undergoing ablation of multiple CRLM at once. Given the natural history of CRLM, it is not uncommon for patients to have multiple metastases, yet the safety and efficacy of multifocal ablation has not been widely reported. We sought to report outcomes of our single institution experience using laparoscopic MWA with or without resection to treat five or more liver metastases. We present the following article in accordance with the
STROBE reporting checklist (available at https://dx.doi. org/10.21037/jgo-21-203).

\section{Methods}

\section{Study design}

After obtaining approval from the University of Minnesota Institutional Review Board, we queried the University of Minnesota Division of Surgical Oncology Liver Surgery Database to identify all patients who underwent ablation of five or more CRLM with or without resection between 2012-2018 at our quaternary referral institution. All patients underwent evaluation by a multidisciplinary liver tumor board consisting of surgical oncologists, interventional radiologists, medical oncologists, hepatologists, transplant surgeons, and radiologists. Lesions that were not surgically resectable and found to be inaccessible for percutaneous ablation by interventional radiology were referred for surgical MWA. Surgical unresectability was determined based on insufficient estimated functional liver remnant, patient comorbidities, or high risk of morbidity of the operation needed to resect both the liver tumor(s) and primary tumor in the colon or rectum. To be considered for surgery, no extrahepatic recurrence (EHR) disease could be present, and all measurable metastatic disease in the liver had to be treatable either by resection or ablation. Tumors $<3 \mathrm{~cm}$ in size located at least $1 \mathrm{~cm}$ from the hilum and central bile ducts were considered candidates for ablation. Out of 93 tumors, one exception was made for a tumor measuring $3.5 \mathrm{~cm}$. Due to the extent of disease in these patients, and the frequent need for additional resectional surgery, none were considered candidates for percutaneous ablation. Staged resections were considered but felt to be unnecessary in the majority of this cohort due to the ability to safely performed combined liver directed therapy with colectomy. Staged resections should be considered in cases where operative morbidity is a concern. For the patients who met inclusion criteria, we obtained baseline characteristics from the electronic medical record. Preand post-operative imaging was reviewed by two blinded independent radiologists subspecialized and fellowship trained in abdominal imaging to determine the maximum size of the lesions and the size of subsequent ablation zones. We also collected and calculated ablation site, intrahepatic, and EHR rates. We classified EHR if disease recurred outside of the liver, intrahepatic recurrence (IHR) if disease recurred within the liver but more than one $\mathrm{cm}$ from any 
Table 1 Demographics and medical characteristics of patients who underwent MWA for colorectal cancer with synchronous liver lesions

\begin{tabular}{lc}
\hline Patient characteristics & Median [range] or $\mathrm{n}[\%]$ \\
\hline Total number of patients & $10[100]$ \\
Age in years at time of diagnosis & $50.5[40-63]$ \\
Age in years at time of operation & $51[41-64]$ \\
Male gender & $7[70]$ \\
Caucasian & $9[90]$ \\
BMl & $26.35[19.1-35.6]$ \\
History of tobacco use & $3[30]$ \\
History of alcohol use & $5[50]$ \\
History of abdominal surgery & $5[50]$ \\
Pre-existing liver disease & 0 \\
\hline
\end{tabular}

MWA, microwave ablation; BMI, body mass index.

Table 2 Clinical and disease characteristics of patients who underwent MWA for colorectal cancer with synchronous liver lesions

\begin{tabular}{lc}
\hline Disease characteristics & Median [range] or $\mathrm{n}[\%]$ \\
\hline Lesion origin & $4[40]$ \\
Colon & $6[60]$ \\
Rectum & $10[100]$ \\
Neoadjuvant chemotherapy & $6[2-13]$ \\
Number of chemotherapy cycles & $2[20]$ \\
Neoadjuvant radiation & 0 \\
Prior liver directed therapy & $3[30]$ \\
Prior colon/rectal resection & $3[2-3]$ \\
Clinical risk score (Fong) & \\
ASA class & 0 \\
I & $5[50]$ \\
II & $5[50]$ \\
III
\end{tabular}

MWA, microwave ablation; ASA, American Society of Anesthesiologists.

previous treatment site, or ablation site recurrence (ASR) if disease recurred within $1 \mathrm{~cm}$ of any previous treatment site.

We identified ten patients with 134 lesions at diagnosis between 2012-2018. Following chemotherapy, 41 lesions disappeared, and 93 lesions were evaluated for treatment.
All patients were treated by a single surgeon with a Certus $1402.45 \mathrm{GHz}$ ablation system (Neuwave Medical, Madison, WI, USA) with laparoscopic ultrasound (BK Medical, Peabody, MA, USA) as previously reported by our group (10). Within 2 weeks of surgery, all patients had repeat MRI to assess for primary treatment failure (incomplete ablation) or missed lesions. Subsequent routine surveillance was performed with CT scan and carcinoembryonic antigen (CEA) evaluations every 3 months for the first 2 years, and then every 6 months thereafter.

\section{Statistical analysis}

The median disease-free survival (DFS) was defined as time between procedure and recurrence. The median overall survival (OS) was defined as time from diagnosis (all patients had synchronous liver lesions) to last follow up or death. Median and range or number and percent were calculated and reported for relevant outcomes.

\section{Ethical statement}

The study was conducted in accordance with the Declaration of Helsinki (as revised in 2013). The study was approved by institutional review board of University of Minnesota (No. 3995) and individual consent for this retrospective analysis was waived.

\section{Results}

\section{Patient demographics}

Ten patients with a total of 93 lesions were identified. All had synchronous liver lesions. Three of the patients were female. The median age at time of diagnosis was 50.5 years. Further patient characteristics are summarized in Table 1.

\section{Disease characteristics}

Disease characteristics, including lesion origin, prior treatment, and American Society of Anesthesiologists (ASA) class are summarized in Table 2. All patients were stage IV at diagnosis and median CEA was 2.2. Table 3 details the tumor characteristics, including number and size of lesions at diagnosis, prior to the operation, and at the time of recurrence. Disappearing liver lesions were not uncommon after neoadjuvant chemotherapy, occurring in $50 \%$ of patients (31\% of all lesions disappeared). 
Table 3 Tumor characteristics on diagnosis, preoperative, postoperative, and recurrence imaging in patients who underwent MWA for colorectal cancer with synchronous liver lesions

\begin{tabular}{lc}
\hline Characteristics & Median [range] \\
\hline Diagnosis & $11[3-41]$ \\
Number of lesions & $1.7[1.1-2.4]$ \\
Largest tumor diameter $(\mathrm{cm})$ & $11[3-41]$ \\
Cumulative tumor diameter $(\mathrm{cm})$ & \\
Preoperative & $9[5-29]$ \\
Number of lesions & $1.2[0.5-2.2]$ \\
Largest tumor diameter $(\mathrm{cm})$ & $5.7[1.6-13.5]$ \\
Cumulative tumor diameter $(\mathrm{cm})$ & \\
Recurrence & $3[1-6]$ \\
Number of lesions & $1.45[0.5-2.5]$ \\
Largest tumor diameter $(\mathrm{cm})$ & $3.95[0.5-6.2]$ \\
Cumulative tumor diameter $(\mathrm{cm})$ &
\end{tabular}

MWA, microwave ablation.

Table 4 Characteristics of the ablation procedure in patients who underwent MWA for colorectal cancer with synchronous liver lesions

\begin{tabular}{lc}
\hline Procedure characteristics & Median [range] or $\mathrm{n}[\%]$ \\
\hline Total number of procedures & 10 \\
Number of open & $4[40]$ \\
Number of laparoscopic & $6[60]$ \\
Number of lesions ablated (per patient) & $7[2-15]$ \\
Number of lesions resected (per patient) & $3[1-5]$ \\
Concurrent partial hepatectomy & $6[60]$ \\
Concurrent bowel/rectal resection & $5[50]$ \\
EBL (mL) & $35[5-400]$ \\
\hline
\end{tabular}

MWA, microwave ablation; EBL, estimated blood loss.

\section{Procedure characteristics}

Table 4 details procedure characteristics, including type of procedure, concurrent procedures, and number of lesions treated. Three of our seven patients did have bowel resection prior to their ablation procedure. Of the seven who did not, five had colon resection at the time of their ablation and two had a liver first approach with complete clinical response to upfront clinical therapy and therefore are on a "Watch and Wait" protocol for their rectal cancer primary.
Table 5 Outcomes of the ablation procedure in patients who underwent MWA for colorectal cancer with synchronous liver lesions

\begin{tabular}{lc}
\hline Outcomes & Median [range] or $\mathrm{n}[\%]$ \\
\hline Hospital length of stay (days) & $4[1-8]$ \\
30-day complications & $4[40]$ \\
30-day mortality & $0[0]$ \\
90-day mortality & $0[0]$ \\
\hline
\end{tabular}

MWA, microwave ablation.

Half of the patients underwent a joint procedure, with the other procedures performed including cholecystectomy, liver resection, colectomy, lysis of adhesions, abdominal perineal resection, colostomy, and appendectomy. In total, 75 lesions were ablated, and 18 lesions were resected over 10 procedures. The median number of lesions ablated per patient was 7 . The total number of lesions treated per patient ranged from 5-18. For patients that had disappearing liver lesions we used ultrasound to assess intraoperatively. In some cases, residual lesions could be identified and treated. However, in other cases, despite the use of intraoperative ultrasound, no lesion could be identified and thus no treatment was performed in that area. These patients were surveilled with imaging every 3 months and any lesion that reappeared was treated immediately either with minimally invasive resection or ablation.

\section{Outcomes}

Peri-operative outcomes, including hospital length of stay, 30-day complications, and mortality are summarized in Table 5. Complications included tumor lysis syndrome, pulmonary embolism, and pleural effusion. All patients received post-operative chemotherapy.

\section{Follow up and survival}

The median follow up was 2.3 years with the longest being 3.8 years. Two patients died of their disease, with both occurring about 2 years after diagnosis and 1.6 years after their ablation procedure. Eight patients had a recurrence, with three patients having local recurrence, six having IHR, and one having EHR. Five patients underwent retreatment with another hepatic directed therapy (ablation, segmental resection, or hepatectomy). The per lesions recurrence rate for ablated lesions was $4 \%$. Primary treatment failure, as defined by incomplete ablation seen on first post-operative 
MRI was seen in zero patients. The median DFS was 7.1 months, and the median OS was 3 years. Patients who had a resection with their ablation had a median OS of 2.8 years compared to 3.1 years in patients who had ablation only. Patients who had a resection with their ablation had a DFS of 5.2 months compared to 15 months in patients who had ablation only. Overall 2 -year survival was $80 \%$. Two patients never had a recurrence and 4 of 5 patients who were retreated remained disease free at last follow-up. In total, $60 \%(6 / 10)$ of patients were disease free at last follow up.

\section{Discussion}

Even though surgical resection is the definitive treatment for CRLM, a large number of lesions are not resectable (5). This is particularly true for patients with multifocal, bilobar metastases, which cannot be resected due to inadequate future liver remnant. In the absence of ablative techniques, most patients would receive palliative chemotherapy alone, which carries an expected survival of 24 months $(11,12)$. Despite having as many as 18 CRLM treated at once, our patients achieved an acceptable OS which compares favorably with palliative chemotherapy alone. Several of our patients did require repeat surgery, and 4/5 who were retreated remained disease free at last follow-up. In light of the extent of disease and high risk of recurrence in this group of patients, we consider our observed OS of 3 years to be quite acceptable.

Other authors have shown that thermal ablation techniques are associated with improved OS in patients who would otherwise only receive systemic therapy. A previous randomized phase 2 trial has shown that liver directed therapy with RFA in addition to palliative chemotherapy for up to 10 hepatic metastases provides improved survival compared to chemotherapy alone (5). This study included 119 patients with up to 10 lesions and no extrahepatic disease and compared a group that received systemic chemotherapy alone to a group that received systemic chemotherapy with radiofrequency ablation with or without resection (5). The combined group had longer median OS at 45.6 compared to 40.5 months for the group that received chemotherapy alone (5). Most notably, based on their survival analysis, ablation appeared to provide a durable survival benefit beyond 9 years in some patients (5). Our study had a per lesion recurrence rate of $4 \%$ which compares favorably to existing data and is likely due to the small median size of tumors considered eligible for ablation.

Due to the small size of this data set, we were unable to identify biologic or tumor related factors that predicted outcomes for our patients. While our in-hospital mortality rate was zero, we did observe post-operative complications related to the large number of metastases treated at once. Most notable was acute renal failure due to tumor lysis syndrome following ablation of 12 lesions (a case report of this complication was immediately published by our group to educate others on its risk) (13). Although thermal ablation has an excellent risk profile, as surgeons treat higher volume CRLM, it is important to inform patients of the potential risk of morbidity with this approach, balanced with the benefits of potential improved survival over palliative chemotherapy.

In this group of patients with as many as 18 CRLM treated, we achieved a very acceptable 2-year survival of $80 \%$ with a median OS of 3 years and $6 / 10$ patients disease free at last follow-up. Given the extent of disease at the time of diagnosis, in the absence of ablation, none of these patients would have been eligible for liver directed surgery, leaving palliative therapy as the only treatment option. We believe that achieving optimal outcomes in this high-risk group is related to multi-disciplinary treatment including systemic chemotherapy (100\% of patients receive up front chemo prior to surgery), careful patient selection, and optimization of MWA surgical techniques. Although much remains to be learned, our data suggests that treating nonresectable CRLM with MWA may be appropriate, even in cases of high-volume tumor burden. Further study will be needed to select those patients who are most likely to benefit from this aggressive approach, however, it is clear that surgeons will play a critical role in defining the utility of ablation in the management of CRLM.

\section{Acknowledgments}

Funding: The Clinical and Translational Science Institute grant (UL1TR002494 from the National Institutes of Health's National Center for Advancing Translational Sciences) supported the use of REDCap software.

\section{Footnote}

Reporting Checklist: The authors have completed the STROBE reporting checklist. Available at https://dx.doi. org/10.21037/jgo-21-203

Data Sharing Statement: Available at https://dx.doi. org/10.21037/jgo-21-203

Peer Review File: Available at https://dx.doi.org/10.21037/ jgo-21-203 
Conflicts of Interest: All authors have completed the ICMJE uniform disclosure form (available at https://dx.doi. org/10.21037/jgo-21-203). CJL reports that he receives grant from Masonic Cancer center for investigation of oncolytic viruses for colorectal cancer therapy. EHJ reports that he is a consultant and receives honoraria from Johnson \& Johnson to teach national and international programs to educate surgeons about the use of microwave ablation. The other authors have no conflicts of interest to declare.

Ethical Statement: The authors are accountable for all aspects of the work in ensuring that questions related to the accuracy or integrity of any part of the work are appropriately investigated and resolved. The study was conducted in accordance with the Declaration of Helsinki (as revised in 2013). The study was approved by institutional review board of University of Minnesota (No. 3995) and individual consent for this retrospective analysis was waived.

Open Access Statement: This is an Open Access article distributed in accordance with the Creative Commons Attribution-NonCommercial-NoDerivs 4.0 International License (CC BY-NC-ND 4.0), which permits the noncommercial replication and distribution of the article with the strict proviso that no changes or edits are made and the original work is properly cited (including links to both the formal publication through the relevant DOI and the license). See: https://creativecommons.org/licenses/by-nc-nd/4.0/.

\section{References}

1. Siegel RL, Miller KD, Goding Sauer A, et al. Colorectal cancer statistics, 2020. CA Cancer J Clin 2020;70:145-64.

2. SEER Cancer Stat Facts: Colorectal Cancer. Bethesda: National Cancer Institute. Available online: https://seer. cancer.gov/statfacts/html/colorect.html

3. Engstrand J, Nilsson H, Strömberg C, et al. Colorectal cancer liver metastases - a population-based study on incidence, management and survival. BMC Cancer 2018;18:78.

Cite this article as: Wothe $\mathrm{JK}, \mathrm{McE}$ achron KR, Marmor S, Ankeny JS, LaRocca CJ, Spilseth B, Schat R, Jensen EH. Surgical microwave ablation for multifocal non-resectable liver metastases: a single institution experience treating five or more liver lesions. J Gastrointest Oncol 2021;12(6):2960-2965. doi: 10.21037/jgo-21-203
4. Zarour LR, Anand S, Billingsley KG, et al. Colorectal cancer liver metastasis: evolving paradigms and future directions. Cell Mol Gastroenterol Hepatol 2017;3:163-73.

5. Ruers T, Van Coevorden F, Punt CJ, et al. Local treatment of unresectable colorectal liver metastases: results of a randomized phase II trial. J Natl Cancer Inst 2017;109:djx015.

6. Correa-Gallego C, Fong Y, Gonen M, et al. A retrospective comparison of microwave ablation vs. radiofrequency ablation for colorectal cancer hepatic metastases. Ann Surg Oncol 2014;21:4278-83.

7. Stättner S, Primavesi F, Yip VS, et al. Evolution of surgical microwave ablation for the treatment of colorectal cancer liver metastasis: review of the literature and a single centre experience. Surg Today 2015;45:407-15.

8. Song P, Sheng L, Sun Y, et al. The clinical utility and outcomes of microwave ablation for colorectal cancer liver metastases. Oncotarget 2017;8:51792-9.

9. Eun HS, Lee BS, Kwon IS, et al. Advantages of laparoscopic radiofrequency ablation over percutaneous radiofrequency ablation in hepatocellular carcinoma. Dig Dis Sci 2017;62:2586-600.

10. McEachron KR, Ankeny JS, Robbins A, et al. Surgical microwave ablation of otherwise non-resectable colorectal cancer liver metastases: expanding opportunities for long term survival. Surg Oncol 2021;36:61-4.

11. Fuchs CS, Marshall J, Mitchell E, et al. Randomized, controlled trial of irinotecan plus infusional, bolus, or oral fluoropyrimidines in first-line treatment of metastatic colorectal cancer: results from the BICC-C Study. J Clin Oncol 2007;25:4779-86.

12. Peeters M, Price TJ, Cervantes A, et al. Randomized phase III study of panitumumab with fluorouracil, leucovorin, and irinotecan (FOLFIRI) compared with FOLFIRI alone as second-line treatment in patients with metastatic colorectal cancer. J Clin Oncol 2010;28:4706-13.

13. Sheka AC, Altman A, Gasparetto A, et al. Tumor lysis syndrome after laparoscopic microwave ablation of colorectal liver metastases. J Gastrointest Cancer 2020;51:631-5. 